\title{
Burden of cardiovascular dysfunction and outcome among term newborns having birth asphyxia
}

\author{
Waqas Shakir ${ }^{1}$, Abdur-Rehman², \\ M. Sohail Arshad ${ }^{3}$, Nazia Fatima ${ }^{4}$
}

\begin{abstract}
Objectives: To find out the burden of cardiovascular dysfunction and outcome among term newborns having birth asphyxia.

Methods: This prospective observational study was conducted at The Department of Neonatology, Children's Hospital and The Institute of Child Health, Multan from August 2020 to March 2021.A total of 171 term newborns having asphyxia were enrolled. Detailed history along with clinical and physical examination were done at the time of admission at Neonatal Intensive Care Unit (NICU). All neonates were followed up for duration of 14 days following birth. Echocardiographic patterns as well as electrocardiography grading were described among neonates with cardiovascular abnormalities.

Results: Out of a total of 171 neonates, there were 94 (55.0\%) male and $77(45.0 \%)$ female. Lowe segment cesarean section was the mode of delivery in $72(42.1 \%)$ while normal vaginal delivery was noted in 99 $(57.9 \%)$. Mean gestational age was noted to be $38.3 \pm 1.8$ weeks. Mean birth weight was calculated to be $2574.10 \pm 122.30$ grams. Cardiovascular dysfunction was noted among $60(35.1 \%)$ neonates as exhibited by the use of inotropes while abnormal ECHO was observed in $52(30.4 \%)$, abnormal ECG in $27(15.8 \%)$ and elevated CK-MB in 31 (18.1\%). A total of $29(17.0 \%)$ asphyxiated neonates died while among 60 asphyxiated neonates with cardiovascular dysfunction, 23 (38.3\%) died and all remaining survived and discharged $(\mathrm{p}<0.0001)$.

Conclusion: Cardiovascular dysfunction among asphyxiated neonates was found to be in high proportion of cases. Cardiovascular dysfunction was noted to have significant association with poor outcome.
\end{abstract}

KEYWORDS: Cardiovascular dysfunction, Birth asphyxia, Inotropes, Echocardiography.

doi: https://doi.org/10.12669/pjms.38.4.5160

How to cite this:

Shakir W, Abdur-Rehman, Arshad MS, Fatima N. Burden of cardiovascular dysfunction and outcome among term newborns having birth asphyxia. Pak J Med Sci. 2022;38(4):883-887. doi: https://doi.org/10.12669/pjms.38.4.5160

This is an Open Access article distributed under the terms of the Creative Commons Attribution License (http://creativecommons.org/licenses/by/3.0), which permits unrestricted use, distribution, and reproduction in any medium, provided the original work is properly cited.

1. Waqas Shakir, FCPS (Pediatrics Medicine), Department of Neonatology,

\section{INTRODUCTION}

2. Abdur Rehman, FCPS

Department of Neonatology,

3. M. Sohail Arshad, FCPS

Department of Pediatric Cardiology,

4. Nazia Fatima, FCPS (Pediatric Medicine), Department of Neonatology,

1-4: Children's Hospital and The Institute of Child Health, Multan, Pakistan.

Correspondence:

Dr. Waqas Shakir

Department of Neonatology,

Children's Hospital and The Institute of Child Health, Multan, Pakistan.

E-mail: dr_waqas226@yahoo.com

* Received for Publication:

August 11, 2021

* Corrected \& Edited:

December 26, 2021

* Accepted for Publication:

January 24, 2022

Perinatal asphyxia is considered to a major cause of neonatal morbidity and mortality while late sequelae of perinatal asphyxia are a cause of major concern especially in developing countries. ${ }^{1,2}$ Perinatal asphyxia commonly affects the brain because of hypoxic-ischemic encephalopathy but other organs or systems are frequently overlooked which also bear the consequences of hypoxic-ischemic insult. Most commonly affected abnormalities of birth asphyxia involve kidneys in about $50 \%$ neonates, central nervous system $28 \%$, cardiovascular $25 \%$ and pulmonary system in $23 \% .^{3}$ This describes

Pak J Med Sci $\quad$ March - April 2022 (Part-II) Vol. 38 No. $4 \quad$ www.pjms.org.pk 883 
that there is involvement of multi-organ dysfunction linked with perinatal asphyxia in the immediate neonatal period.

Myocardium of neonates is thought to be resistant to hypoxia ${ }^{4}$ but cardiac failure is one of the most important manifestations of myocardial dysfunction among cases having perinatal asphyxia. ${ }^{5}$ Although prevalence of severe cardiac damage is not high but relatively less severe manifestations involving heart might be frequent among neonates with asphyxia. ${ }^{6}$ In the past, murmur suggesting Atrioventricular insufficiency, electrocardiographic abnormality depicting myocardial ischemia, cardiogenic shock, hypotension, functional tricuspid incompetence or arrhythmia have been found to be frequent cardiovascular complications among neonates with asphyxia. ${ }^{7}$

In Pakistan, no study has specifically aimed at finding out cardiovascular abnormalities and outcome in birth asphyxia but data from India suggest that significant proportion of neonates $(32 \%)$ were having cardiovascular dysfunction while neonates with cardiovascular dysfunction had significantly increased risk of poor outcome. ${ }^{8}$ This study was aimed at finding out burden of cardiovascular dysfunction and outcome in birth asphyxia. The findings of this study were thought to provide useful insights about the possible burden and types of cardiovascular involvement among neonates born with asphyxia.

\section{METHODS}

This was a prospective observational study conducted at department of neonatology, Children's Hospital and The Institute of Child Health, Multan from August 2020 to March 2021. Approval from Institutional Ethical Committee was taken (Ref\#ERC/162, Dated: 06/04/2020). Written consent was sought from parents/guardians of all study participants.

A sample size of 171 cases was calculated considering 95\% confidence interval, margin of error as $7 \%$ and incidence of cardiovascular abnormalities as $32 \%$ in birth asphyxia. A total of 171 newborns having asphyxia as Apgar score less than or equal to seven at five minutes with or without umbilical cord arterial $\mathrm{pH}$ below 7.2 at the time of birth or/and those who needed $\geq 1$ minute of positive pressure ventilation prior to sustained respiration or required mechanical ventilation at the time of birth were enrolled. ${ }^{8}$ All children were enrolled within 24 hours following birth and had gestational age between 37 to 42 weeks. Children having congenital anomalies or those who were suspected to have early-onset sepsis were not included. Children leaving against medical advice or whose parents/guardians did not give consent to be part of this study were also excluded.

Detailed history along with clinical and physical examination were done at the time of admission at Neonatal Intensive Care Unit (NICU). All neonates were followed up for duration of 14 days following birth. Gestational age, mode of delivery and indications for any intervention (if any) were recorded. Any complications before or during the labor were recorded accordingly. Postnatal history like birth asphyxia, resuscitation measures adopted at the time of birth along with Apgar score at $1^{\text {st }}$ and $5^{\text {th }}$ minute was noted. Echocardiography was done while each neonate was assessed for the existence of heart murmur, dysrhythmias, cyanosis, rise respiratory distress with systemic hypotension or signs of shock three seconds) were noted. Inotropes were initiated if neonates continued to have signs of systemic hypoperfusion even after fluid boluses up to 40 $\mathrm{ml}$ per $\mathrm{kg}$. Transient myocardial ischemia was identified with the help of 12-lead serial ECG in the $1^{\text {st }}$ three days of admission. Grade- 1 ECG changes were labeled as flat/inverted T-waves on one or two limb leads AVR. Grad-2 was described as flat/inverted T-waves in $\geq 3$ leads except AVR. Grad-3 was labeled as flat/inverted T-waves in $\geq 3$ leads and either ST depression or elevation more than $2-\mathrm{mm}$ in at least two chest leads or more than $1 \mathrm{~mm}$ in at least 2 standard leads, or a Q-wave abnormality of more than 0.02 seconds or amplitude above $25 \%$ of R-wave in one anterior or three related chest leads. Grade- 4 was labeled as presence or classical segmental infarction with abnormal Q-wave and markedly elevated ST segment or complete left bundle branch block. Creatinine kinase-MB (CK-MB) isoenzyme levels were noted at eight hours and 24 hours following admission while values above $92.6 \mathrm{U} / \mathrm{L}$ at 8 hours and $60 \mathrm{U} / \mathrm{L}$ at 24 hours were considered as high. Among neonates who had persistent murmurs and/or abnormal findings, $\mathrm{ECHO}$ was done.

SPSS version 26.0 was used for data analysis. Qualitative variables like gender, mode of delivery, use of inotropes and presence of cardiovascular involvement were represented as frequency and percentages. Echocardiographic patterns as well as electrocardiography grading were described among neonates with cardiovascular 
Table-I: Maternal and Fetal Characteristics of Study Participants $(n=171)$.

\begin{tabular}{llc}
\hline Characteristics & Number (\%) \\
\hline \multirow{3}{*}{ Gender } & Male & $94(55.0 \%)$ \\
& Female & $77(45.0 \%)$ \\
Mode of & Cesarean Section & $72(42.1 \%)$ \\
Deliver & Vaginal Delivery & $99(57.9 \%)$ \\
Gestational & $37-39$ & $128(74.9 \%)$ \\
Age & $40-42$ & $43(25.1 \%)$ \\
Birth Weight & $<2500$ & $39(22.8 \%)$ \\
(grams) & $\geq 2500$ & $132(77.2 \%)$ \\
\hline
\end{tabular}

abnormalities. Poor outcome (death) was compared using chi square test among asphyxiated neonates with and without cardiovascular dysfunction. $\mathrm{P}$ value less than 0.05 was considered as significant. A special proforma was made to record all study information.

\section{RESULTS}

Out of a total of 171 neonates, there were 94 $(55.0 \%)$ male and $77(45.0 \%)$ female. Lowe segment cesarean section was the mode of delivery in 72 $(42.1 \%)$ while normal vaginal delivery was noted in $99(57.9 \%)$. Mean gestational age was noted to be $38.3 \pm 1.8$ weeks. Mean birth weight was calculated to be $2574.10 \pm 122.30$ grams. Table-I is showing maternal and fetal characteristics of study participants.

Cardiovascular dysfunction was noted among 60 $(35.1 \%)$ neonates as exhibited by the use of inotropes while abnormal ECHO was observed in $52(30.4 \%)$, abnormal ECG in $27(15.8 \%)$ and elevated CK-MB in 31 (18.1\%). Fig.1 is showing cardiovascular system involvement noted among asphyxiated neonates.

Use of inotropes was noted to be among 60 $(35.1 \%)$ neonates at day-1 of admission while it

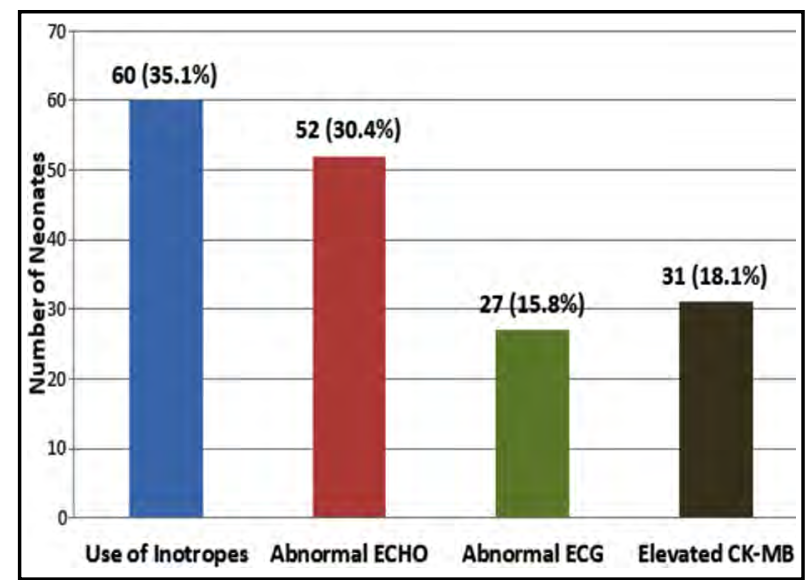

Fig.1: Cardiovascular Involvement among Asphyxiated Neonates $(\mathrm{n}=171)$.

was in $41(24.0 \%)$ at day-3, $14(8.2 \%)$ at day-7 while none of the neonates required inotropes at day-14. Among 27 neonates with abnormal ECG, 18 were having Grade-1 ECG changes, five had Grade-2 and Grade- 4 as Grade- 4 changes. Persistent pulmonary hypertension was found to be the commonest echocardiographic abnormality observed in 25 $(14.6 \%)$ neonates. Distribution and patterns of echocardiographic findings among asphyxiated neonates is shown in Table-II.

A total of $29(17.0 \%)$ asphyxiated neonates died while among 60 asphyxiated neonates with cardiovascular dysfunction, $23(38.3 \%)$ died and all remaining survived and discharged $(\mathrm{p}<0.0001)$. Table-III

\section{DISCUSSION}

Perinatal asphyxia is considered to be a major yet preventable cause of neonatal mortality especially in developing countries. ${ }^{1}$ In the present study, cardiovascular dysfunction as need for the use of inotropes was noted in $35.1 \%$ asphyxiated neonates. De Dios JG et al from Spain reported cardiovascular manifestations among $19.8 \%$ of asphyxiated newborns which is lower than what

Table-II: Echocardiographic Findings among Asphyxiated Neonates.

\begin{tabular}{lc}
\hline Echocardiographic Findings & Number (\%) \\
\hline Persistent Pulmonary Hypertension & $25(14.6 \%)$ \\
Moderate Patent Ductus Arteriosus & $16(9.4 \%)$ \\
Moderate Patent Ductus Arteriosus + Patent Foramen Ovale & $5(2.9 \%)$ \\
Moderate Patent Ductus Arteriosus + Patent Foramen Ovale + Moderate Tricuspid Regurgitation & $3(1.8 \%)$ \\
Ventricular Septal Defect & $3(1.8 \%)$ \\
\hline
\end{tabular}


Table-III: Distribution of Mortality with respect to Cardiovascular Dysfunction.

\begin{tabular}{lccc}
\hline & \multicolumn{2}{c}{ Cardiovascular Dysfunction } & \\
\cline { 2 - 3 } Mortality & Yes $(n=60)$ & No $(n=121)$ & \\
\hline Yes & $23(38.3 \%)$ & $6(5.0 \%)$ & \\
No & $27(61.7 \%)$ & $115(95.0 \%)$ & $<0.0001$ \\
\hline
\end{tabular}

we found in the present study. ${ }^{9}$ Shah et al found predominant majority of asphyxiated neonates $(62 \%)$ who needed inotropes. ${ }^{10}$ Hankins and colleagues also revealed that $61 \%$ of the asphyxiated neonates needed inotropes. ${ }^{11}$ On the other hand Martin-Ancel et al revealed that only $4 \%$ of the asphyxiated neonates needed inotropes..$^{12}$ All these variations could be due to different composition of study sample involving variable number of neonates with different definitions of severity levels of perinatal asphyxia. ${ }^{13}$ Researchers have highlighted significant presence of cardiovascular dysfunction that is secondary to neonatal asphyxia but no studies have been done to find out longterm cardiovascular outcomes. ${ }^{14}$ Only some researchers have highlighted persistent pulmonary hypertension to be long-term cardiovascular abnormality among asphyxiated neonates. ${ }^{15}$

In this study we found that abnormal ECG was found among $15.8 \%$ asphyxiated neonates whereas among these neonates, $66.7 \%$ were having Grade-1 ECG changes. Some other researchers have found a higher proportion of neonates $(76.7 \%)$ with abnormal ECG findings. Rajakumar et al reveled $73.3 \%$ of the asphyxiated neonates to report ECG changes. The ECG abnormalities could be indicating myocardial ischemia secondary to birth asphyxia. ${ }^{16}$ Kumar PS from India recorded ECG abnormalities among $46.6 \%$ of the asphyxiated neonates. ${ }^{17}$

Elevated CK-MB was noted among 18.1\% asphyxiated neonates. This is pretty consistent to what has previously been found by Singh et al where they revealed $14.5 \%$ of the asphyxiated neonates to have elevated CK-MB. ${ }^{8}$ Hankins et al reported $17 \%$ of the asphyxiated neonates to have elevation of CK-MB levels. ${ }^{11}$ Significance of CK$\mathrm{MB}$ as a possible indicator of myocardial injury among neonates with asphyxia still needs further evaluation in the future studies.

In the present study, $30.4 \%$ asphyxiated neonates were found to have abnormal ECHO findings while most abnormality was persistent pulmonary hypertension followed by moderate patent ductus arteriosus. Linkage between meconium aspiration and persistent pulmonary hypertension has been reported in the literature ${ }^{18}$ but we could not report presence of meconium aspiration among present group of study cases due to limitation of our study protocol. Similarly, raised pulmonary pressure could be a contributing factor to hemodynamic instability. A study from Iran reported $62 \%$ of the asphyxiated neonates to have patent ductus arteriosus. ${ }^{19}$ Some studies have indicated patent arterial duct to be a factor responsible for causing cardiovascular dysfunction and persistent pulmonary hypertension. ${ }^{20,21}$

In the present research, we found that among 60 asphyxiated neonates with cardiovascular dysfunction, $23(38.3 \%)$ died. Singh et al reported mortality among $42 \%$ of neonates with cardiovascular dysfunction while Shah et al found $64 \%$ of asphyxiated neonates with cardiovascular abnormalities to report adverse outcomes or death. ${ }^{8}$ Among asphyxiated neonates, high index of suspicion should be made for the early detection of cardiovascular abnormalities as timely identification and management can lead to improved outcome.Being the $1^{\text {st }}$ study from Pakistan that looked into cardiovascular dysfunctions among asphyxiated neonates is one of the major strength of this study.

Limitations of the study: As this was a single study with no comparator or control groups, further multicenter studies with comparative designs should be conducted. We did not plan to monitor the surviving neonates for possible longterm outcomes. More studies are required to find out correlation of different stages of asphyxia with different cardiovascular manifestations among asphyxiated neonates.

\section{CONCLUSION}

Cardiovascular dysfunction among asphyxiated neonates was found to be in high proportion of cases. Cardiovascular dysfunction was noted to have significant association with poor outcome. Early screening as well as identification of cardiovascular system among asphyxiated neonates might help decreasing the burden of morbidity and mortality related to cardiovascular dysfunction among asphyxiated neonates.

Acknowledgement: Thanks to M. Aamir (Research Consultant, Bahawalpur) for the volunteer support in statistical analysis. 


\section{Conflict of Interest: None}

\section{Sponsorship or Source of Funding: None.}

\section{REFERENCES}

1. Arshad MS, Adnan M, Anwar-ul-Haq HM, Zulqarnain A. Postnatal causes and severity of persistent pulmonary Hypertension of Newborn. Pak J Med Sci. 2021;37(5):13871391. doi: 10.12669 / pjms.37.5.2218

2. Samad N, Farooq S, Hafeez K, Maryam M, Rafi MA. Analysis of consequences of birth asphyxia in infants: A regional study in Southern Punjab, Pakistan. J Coll Phys Surg Pak. 2016;26(12):950-953.

3. Tanzil WL, Wilar R, Mantik MF, Umboh A, Tatura SN. Comparison of urine neutrophil gelatinase-associated lipocalin to serum creatinine to assess kidney function in neonatal asphyxia. Paediatr Indones. 2016;56:356-359. doi: 10.14238/pi56.4.2016.356-9

4. Baker JE. Oxidative stress and adaptation of the infant heart to hypoxia and ischemia. Antioxid Redox Signal. 2004;6(2):423-429. doi: 10.1089/152308604322899495

5. Rowe RD, Izukawa T, Mulholland HC, Bloom KR, Cook DH, Swyer PR, et al. Nonstructural heart disease in the newborn. Observations during one year in a perinatal service. Arch Dis Child 1978;53:726-730.

6. Ranjit MS. Cardiac abnormalities in birth asphyxia. Indian J Pediatr. 2000;67(3-Suppl):S26-S29.

7. Dijxhoorn MJ, Visser GH, Fidler VJ, Touwen BC, Huisjes HJ. Apgar score, meconium and acidaemia at birth in relation to neonatal neurological morbidity in term infants. Br J Obstet Gynaecol 1986;93:217-922. doi: 10.1111/j.1471-0528.1986. tb07896.x

8. Singh V, Vohra R, Bansal M. Cardiovascular involvement in birth asphyxia. J Clin Neonatol 2018;7:20-24. doi: 10.4103/ jcn.JCN_80_17

9. de Dios JG, Benavent M, Iglesias C, Sanchez Y. Importancia clínica y pronóstica de las manifestaciones cardiovasculares en la asfixia perinatal [Clinical and prognostic value of cardiovascular symptoms in perinatal asphyxia]. An Esp Pediatr. 1997;47(3):289-294.

10. Shah P, Riphagen S, Beyene J, Perlman M. Multiorgan dysfunction in infants with post-asphyxial hypoxic-ischaemic encephalopathy. Arch Dis Child Fetal Neonatal Ed 2004;89:F152-5. doi: 10.1136/adc.2002.023093

11. Hankins GD, Koen S, Gei AF, Lopez SM, Van Hook JW, Anderson GD. Neonatal organ system injury in acute birth asphyxia sufficient to result in neonatal encephalopathy. Obstet Gynecol. 2002 May;99(5 Pt 1):688-91. doi: 10.1016/ s0029-7844(02)01959-2

12. Martin-Ancel A, García-Alix A, Gaya F, Cabanas F, Burgueros M, Quero J, et al. Multiple organ involvement in perinatal asphyxia. J Pediatr 1995;127:786-93. doi: 10.1016/ s0022-3476(95)70174-5
13. Polglase GR, Centre TR, Ong T, Centre TR, Hillman NH. Cardiovascular alterations and multi organ dysfunction after birth asphyxia. Clin Perinatol. 2017;43(3):469-483. doi: 10.1016/j.clp.2016.04.006

14. Popescu MR, Panaitescu AM, Pavel B, Zagrean L, Peltecu G, Zagrean AM. Getting an Early Start in Understanding Perinatal Asphyxia Impact on the Cardiovascular System. Front Pediatr. 2020;8:68. doi: 10.3389/fped.2020.00068

15. Rosenzweig EB, Abman SH, Adatia I, Beghetti M, Bonnet D, HaworthS, etal. .Paediatric pulmonary arterial hypertension: updates on definition, classification, diagnostics and management. Eur Respir J. 2019;53(1):1801916. doi: 10.1183/13993003.01916-2018

16. Rajakumar PS, Bhat VB, Sridhar MG, Balachander J, Konar BC, Narayanan P, et al. Electrocardiographic and echocardiographic changes in perinatal asphyxia. Indian J Pediatr. 2009;76(3):261-4. doi: 10.1007/s12098-008-0221-4

17. Kumar PS, Arasan GD. A biochemical profile of cardiac involvement in perinatal asphyxia. Int J Contemp Pediatr. 2018;5(2):328-333. doi: 10.18203/2349-3291.ijcp20180033

18. Choudhary M, Meena MK, Chhangani N, Sharma D, Choudhary JS, Choudhary SK. To study prevalence of persistent pulmonary hypertension in newborn with meconium aspiration syndrome in western Rajasthan, India: A prospective observational study. J Matern Fetal Neonatal Med. 2016;29(2):324-327. doi: 10.3109/14767058.2014.1000296

19. Shahidi M, Evazi G, Afkhamzadeh A. Echocardiographic evaluation of cardiovascular complications after birth asphyxia in term neonates. Pak J Med Sci. 2017;33(5):12201224. doi: 10.12669 / pjms.335.12849

20. Capozzi G, Santoro G. Patent ductus arteriosus: patho-physiology, hemodynamic effects and clinical complications. J Matern Fetal Neonatal Med. 2011;24(Suppl 1):15-16. doi: 10.3109/14767058.2011.607564

21. Lin YC, Huang HR, Lien R, Yang PH, Su WJ, Chung HT, et al. Management of patent ductus arteriosus in term or nearterm neonates with respiratory distress. Pediatr Neonatol. 2010;51(3):160-165. doi: 10.1016/s1875-9572(10)60030-7

\section{Authors' Contribution:}

WS: Data Collection, Drafting, Responsible for Data's Integrity

AR: Methodology, Supervision, Proof Reading

MSA: Review of Literature, Data Analysis

NF: Data Interpretation, References. 